\title{
Utilização de planejamento fatorial para a determinação da capacidade antioxidante e doseamento de flavonoides totais em Verbena minutiflora Briq. ex Moldenke (gervai)
}

PELOI, K.E.'; BOVO, F.2; MESSIAS-REASON, I.J.2; PEREZ, E. ${ }^{3}$

1Universidade Estadual de Maringá. Avenida Colombo, 5790- Jardim Universitário, Maringá - PR, CEP: 87020900. 2Universidade Federal do Paraná, Departamento de Patologia médica Hospital de Clínicas da UFPR- Rua Padre Camargo, 280-Alto da Glória- Curitiba, PR - CEP: 80060240. ${ }^{3}$ Universidade Estadual do Centro Oeste. - Rua Simeão Camargo Varela de Sá, 03, CEDETEG-Vila Carli- Guarapuava, PR. CEP: 85040-080. *Autor para correspondência: elisaevangeline@gmail.com

RESUMO:Verbena minutiflora Briq. ex Moldenke (gervai) tem seu uso medicinal relatado popularmente para tratamento de doenças hepáticas, diarreia e outros problemas de saúde. Entretanto, pouco se conhece a respeito de seus componentes químicos e estudos que comprovem suas propriedades medicinais são escassos. $\mathrm{O}$ objetivo desse estudo foi avaliar a composição química dos extratos aquosos e etanólicos de flores de $V$. minutiflora e otimizar processos de obtenção de extratos com maiores capacidades antioxidantes e maiores concentrações de flavonoides. O método de extração foi desenhado por planejamento fatorial, onde as variáveis para a determinação da capacidade antioxidante foram: $\mathrm{pH}$, extração líquida, método e tempo de extração. Para a determinação de flavonoides totais as variáveis avaliadas por planejamento fatorial foram: concentração de hexametilenotetramina, tipo de ácido, volume de ácido e tempo de aquecimento. Os resultados das análises químicas dos extratos mostraram: aminogrupos, taninos e ácidos fixos (extrato aquoso) aminogrupos, flavonoides, triterpenos, esteroides, alcaloides e cumarinas (extrado hidroetanólico). Os resultados dos planejamentos fatoriais mostraram que o melhor método de extração para a capacidade antioxidante foi o

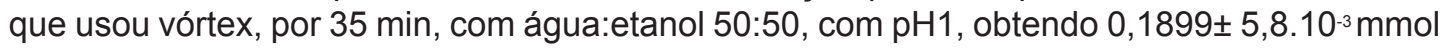
expressos em ácido ascórbico g-1 nos extratos de $V$. minutiflora. Enquanto, para as dosagens de flavonoides totais as variáveis significantes foram: tipo de ácido e volume de ácido. A melhor extração obtida foi: 6,748 . 10-2 $\pm 2,085$ 10-3\% expressos em quercetina. Os resultados mostraram que o planejamento fatorial é uma importante ferramenta para a otimização de extração de componentes químicos em produtos naturais.

Palavras chaves: espectrofotometria, otimização multivariada, farmacognosia.

\begin{abstract}
The use of factorial design for determining the antioxidant capacity and dosing the total flavonoids in Verbena minutiflora Briq. Ex Moldenke (Gervai). Verbena minutiflora Briq. ex Moldenke (gervai) has its popular use reported for liver disorders treatments, diarrhea, and other health problems. However, little is known about its chemical components and studies that proves its medicinal properties are rare. The aim of this study was to evaluate the chemical composition of aqueous and ethanolic extracts from flowers of $V$. minutiflora and to optimize processes to obtain extracts with higher antioxidant capacity and greater concentration of flavonoids. The methods of extraction were designed by factorial planning, where the variables to determine the antioxidant capacity were: $\mathrm{pH}$; extraction liquid; method and extraction time. To determinate the total flavonoids the variables evaluated by factorial design were: concentration of hexamethylenetetramine; type of acid; volume of acid and warming time. The results of chemical analysis of the extracts showed: amino groups, tannins and fixed acids (aqueous extract) amino groups, flavonoids, triterpenes, steroids, alkaloids and coumarins (hydroalcoholic extract). The factorial designs results showed that the best extraction method for the antioxidant capacity was the one that uses vortex, for $35 \mathrm{~min}$, with water: ethanol 50:50, at $\mathrm{pH} 1$, getting 0,1899 \pm $5,8.10^{-3} \mathrm{mmol}$ expressed in ascorbic acid g-1 in extracts of $V$. minutiflora. While, for dosages of total flavonoids the significant variables were the type of acid and volume of acid. The best extraction obtained was: $6,748.10^{-2} \pm 2,08510^{-3} \%$ expressed in quercetin. These data showed
\end{abstract}

Rev. Bras. PI. Med., Campinas, v.18, n.1, p.1-8, 2016. 
that the factorial design is an important tool in optimizing the extraction of chemical components in natural products.

Keywords: spectrophotometry, multivariate optimization, pharmacognosy.

\section{INTRODUÇÃO}

A utilização racional de plantas medicinais com fins terapêuticos pode constituir um importante fator de desenvolvimento da economia local, dos órgãos e instituições envolvidas com os setores da saúde de países em desenvolvimento (Maciel et al., 2002; Lameira et al., 2008).

O Brasil detém a maior diversidade biológica do mundo, conta com uma rica flora, a qual tem despertado interesse de pesquisadores internacionais, especialmente na área medicinal (Souza \& Felfili, 2006). Porém, apesar da rica flora e da sabida importância do conhecimento sobre plantas, pesquisas brasileiras a respeito dos componentes químicos e das propriedades dessa flora nacional, ainda são escassas.

A espécie vegetal $V$. minutiflora Briq. ex Moldenke, Verbenaceae, popularmente conhecida como Gervão, é facilmente encontrada em terrenos baldios, beiras de estradas e pastagens (Morcelle et al.,2012). Pouco se sabe a respeito de suas propriedades medicinais. Seu gênero, Verbena, é conhecido popularmente por ser hepatoprotetor, anti-inflamatório e antidiarreico, dentre outras propriedades medicinais (Morcelle et al., 2012). Estudos sobre seus componentes químicos, metabólitos primários e secundários são escassos. Assim, trabalhos que caracterizem quimicamente e investiguem possíveis propriedades medicinais desta espécie mostram-se de grande importância.

A utilização da ferramenta de planejamento fatorial (PF) em estudos que abordam muitas variáveis, como é o caso de otimizações de componentes químicos em produtos naturais, é muito importante, pois torna possível o planejamento e a realização de uma quantidade reduzida de experimentos, o que economiza tempo e recursos financeiros, itens importantes em laboratórios de pesquisas (Silva et al., 2006).

Apesar da relatada relevância das ferramentas de análises multivariadas, não há na literatura, relatos da utilização de PF na otimização da extração de componentes químicos envolvendo a espécie $V$. minutiflora.

Este trabalho teve como objetivo utilizar o P.F., como metodologia para a otimização do processo de extração das flores do gervão visando a: 1) obtenção de extratos com maior capacidade antioxidante e; 2) obtenção de extratos ricos em flavonoides.

\section{MATERIAL E MÉTODOS Material Vegetal}

Os exemplares de V. minutiflora foram coletados em Guarapuava-PR (dezembro de 2009), identificados ( $n^{\circ} 331054$, Museu Botânico Municipal de Curitiba-PR) e secos à sombra com intensa claridade. As flores foram trituradas em moedor de copo. O pó foi tamisado (tamis ABNT 60) e as partículas com granulometria inferior ou igual a $0,250 \mathrm{~mm}$ de tamanho foram utilizadas em todos os experimentos.

\section{Preparo e análises dos extratos}

Foi preparado um extrato aquoso a $20 \%$ (p. $\left.\mathrm{v}^{-1}\right)$, para isto, misturou-se o material vegetal com o solvente (água destilada) em banho-maria a $60^{\circ} \mathrm{C}$ por $2 \mathrm{~h}$, em seguida, o material obtido foi filtrado e armazenado a $-8^{\circ} \mathrm{C}$.

Preparou-se também um extrato hidroalcoólico a $20 \%$ (p. $\left.v^{-1}\right)$ utilizando-se como solvente o etanol $70 \%$ (v.v-1), por maceração, durante sete dias e, posteriormente o material obtido foi filtrado e armazenado a $4^{\circ} \mathrm{C}$.

A partir desses extratos foram realizadas pesquisas qualitativas de metabólitos secundários através de testes fitoquímicos (Matos, 1997). Além disso, foram determinadas as características organolépticas e pHs dos extratos. Foi realizada ainda, a determinação de água pelo método gravimétrico, em método adaptado da Farmacopeia brasileira (Brasil, 2010a), onde utilizou-se 0,5 g do pó $(n=3)$ das flores tamisadas, dessecando-as em estufa a $105^{\circ} \mathrm{C}$ até peso constante e após isso, o teor de água calculado.

\section{Determinação da capacidade antioxidante}

A determinação da capacidade antioxidante foi realizada segundo Prieto et al (1999) com a ajuda do planejamento fatorial. Para isso, foi utilizada uma solução reagente oxidante (SRO) composta de $0,6 \mathrm{M} \mathrm{H}_{2} \mathrm{SO}_{4}, 28 \mathrm{mM}$ de $\mathrm{Na}_{3} \mathrm{PO}_{4} .12 \mathrm{H}_{2} \mathrm{O}$ e $4 \mathrm{mM}$ de molibdato de amônio.

\section{Curva analítica para o ensaio da capacidade antioxidante}

Para a construção da curva analítica foi utilizada uma solução-estoque de ácido ascórbico (padrão externo) $3,577 \mathrm{mM}$ e volumes variados dessa solução foram tratados com $1,5 \mathrm{~mL}$ de SRO, aquecidos em banho-maria a $95^{\circ} \mathrm{C}$ por $90 \mathrm{~min}$.

Rev. Bras. PI. Med., Campinas, v.18, n.1, p.1-8, 2016. 
As soluções resultantes foram centrifugadas por 5 minutos a $8700 \times g$ e a seguir, fez-se as leituras das absorvâncias em 695 nm, para a obtenção da equação da reta, intervalo e coeficiente de correlação linear $\left(R^{2}\right)$.

\section{Preparo de amostra}

Desenhou-se um planejamento fatorial 24-1 (ponto central $n=5$ ). Na otimização da extração foram extraídos $0,040 \mathrm{~g}$ do pó das flores tamisadas com diferentes líquidos extratores (água ou água etanol 50:50, $2 \mathrm{~mL}$ ), em diferentes $\mathrm{pHs}$ ( 1 ou 5 , com $\mathrm{HCl} \mathrm{PA),} \mathrm{com} \mathrm{diferentes} \mathrm{métodos} \mathrm{de} \mathrm{extração}$ (sonicação ou vórtex) e por tempos diferentes de extração (5 ou 15 min), conforme a matriz de contrastes (Tabela. 1).

Após a realização do ensaios desenhados por PF, as amostras foram centrifugadas por 5 min a $17400 \times$ g. Alíquotas $(50 \mu \mathrm{L})$ dos sobrenadantes foram acrescentadas a $1,5 \mathrm{~mL}$ de $\mathrm{SRO}$ (Prieto et al., 1999). As misturas resultantes foram aquecidas em banho-maria por $90 \mathrm{~min}$ a $95^{\circ} \mathrm{C}$. Após resfriamento, as misturas foram novamente centrifugadas por 5 min a 8700 x $g$ e as absorvâncias dos sobrenadantes foram lidas em $695 \mathrm{~nm}$. Os brancos do ensaio constaram dos respectivos líquidos extratores acrescidos de SRO. As absorvâncias foram contrastadas com a curva analítica, com a expressão dos resultados em mmol.g-1 de ácido L-ascórbico em flores da planta.

Os resultados foram analisados através de planilhas de quimiometria (Teófilo \& Ferreira., 2006) com $\alpha=0,05$ ou, alternativamente, obteve-se o desvio do efeito (DE), onde $D E=t$.dp sendo " $t$ " o valor da distribuição $t$ de Student com $\alpha=0,05$, com n-1 graus de liberdade e "dp" o desvio padrão dos valores do ponto central, contrastando-se com o efeito calculado para cada variável, onde:

$$
e f=\frac{\sum_{i=1}^{n / 2} y_{i(+)}-\sum_{i=1}^{n / 2} y_{i(-)}}{n / 2}
$$

sendo "ef" o efeito calculado para cada variável ou interações pela diferença entre as médias das observações no nível mais $\left(y_{i(+)}\right)$ e as médias das observações no nível menos $\left(y_{i--}\right)$, para $n$ ensaios e $y_{i}$ observações individuais (Teófilo \& Ferreira., 2006).

Com as variáveis significativas e os efeitos de $2^{a}$ ordem obtidos foram realizados outros planejamentos fatoriais (ponto central $\mathrm{n}=5$ ). Posteriormente, a otimização foi finalizada com método univariado e de posse da melhor metodologia de preparo de amostra, determinou-se a capacidade antioxidante das flores $(n=6)$.

\section{Totais \\ Determinação do teor de Flavonoides \\ Para o doseamento de flavonoides totais,} utilizou-se como base um método farmacopeico (Farmacopeia brasileira, 2001).

\section{Curva analítica}

Para a realização da curva analítica utilizada na determinação de flavonoides totais foi preparada uma solução estoque 1,0 $\mu \mathrm{g} \cdot \mathrm{mL}^{-1}$ de quercetina/ metanol PA, sendo a quercetina utilizada, neste ensaio, como padrão externo. Diferentes volumes dessa solução foram adicionados a $0,4 \mathrm{~mL}$ de "Solução Reagente de Flavonoides" (SRF- solução composta de $\mathrm{AlCl}_{3} \cdot 6 \mathrm{H}_{2} \mathrm{O}$ em ácido acético/metanol $5 \%(\mathrm{v} / \mathrm{v}) 8 \mathrm{mM})$. À cada amostra obtida da mistura da solução padrão com SRF, foi acrescentado ácido acético/metanol $5 \%(\mathrm{v} / \mathrm{v})$ qsp (quantidade suficiente para) $10 \mathrm{~mL}$. Após $30 \mathrm{~min}$, as soluções resultantes foram lidas em $425 \mathrm{~nm}$, obtendo-se a equação linear da curva, $R^{2}$ e intervalo de trabalho (Teófilo \& Ferreira., 2006).

TABELA 1. Matriz de contrastes para a determinação da capacidade antioxidante, como planejamento fatorial 24-1 com ponto central

\begin{tabular}{ccccc}
\hline Amostras & $\mathrm{pH}$ & Líquido extrator & Método de extração & Tempo de extração \\
\hline $\mathbf{1}$ & $-(\mathrm{pH} 1)$ & - (água) & $-($ vórtex $)$ & $-(5 \mathrm{~min})$ \\
2 & $+(\mathrm{pH} 5)$ & - & - & $+(15 \mathrm{~min})$ \\
$\mathbf{3}$ & - & + (água:etanol 50:50) & - & + \\
$\mathbf{4}$ & + & + & - & - \\
$\mathbf{5}$ & - & - & + (sonicador $)$ & - \\
$\mathbf{6}$ & + & - & + & - \\
7 & - & + & + & + \\
$\mathbf{8}$ & + & + & Água:etanol 75:25 & Vórtex (V) e Sonicador (S) 5 min vórtex e 5 min sonicador \\
\hline
\end{tabular}

Rev. Bras. PI. Med., Campinas, v.18, n.1, p.1-8, 2016. 


\section{Preparo de amostra}

O preparo das amostras utilizadas no doseamento de flavonoides foi desenhado por um planejamento fatorial fracionado $2^{4-1} \mathrm{com}$ ponto central $(n=3)$, com $\alpha=0,05$, conforme a matriz de contrastes (Tabela .2), onde as variáveis avaliadas foram: quantidade de solução de hexametilenotetramina (HMT) PA a 0,5\% (1 ou 2 $\mathrm{mL})$, tipo de ácido $\left(\mathrm{HCl}\right.$ ou $\left.\mathrm{H}_{2} \mathrm{SO}_{4}\right)$, volume de ácido ( 2 ou $4 \mathrm{~mL}$ ) e tempo de aquecimento (15 min mais $5 \mathrm{~min}$ ou $30 \mathrm{~min}$ mais $10 \mathrm{~min}$ ).

A massa de $0,4 \mathrm{~g}$ da droga foi extraída com $20 \mathrm{~mL}$ de acetona PA, $0,5 \%(\mathrm{p} / \mathrm{v})$ e $\mathrm{HCl}$ ou $\mathrm{H}_{2} \mathrm{SO}_{4}-$ nos volumes definidos pelo planejamento. Essas misturas foram aquecidas individulamente em banho-maria sob refluxo pelo tempo determinado no P.F. Após filtração em algodão o filtrado foi reservando. $O$ algodão e os resíduos foram reextraídos em refluxo com $15 \mathrm{~mL}$ de acetona, pelos diferentes tempos definidos no planejamento, filtrando-se em seguida, sendo esse procedimento repetido três vezes. Os filtrados foram reunidos, resfriados e acetona foi acrescentada qsp $50 \mathrm{~mL}$. Adicionou-se uma alíquota de $10 \mathrm{~mL}$ dessa solução a $10 \mathrm{~mL}$ de água e $5 \mathrm{~mL}$ de acetato de etila e em funil de separação, a fase orgânica foi coletada. Repetiu-se a extração 3 vezes com $5 \mathrm{~mL}$ de acetato de etila e todas as fases orgânicas foram reunidas, lavando-as com duas porções de $25 \mathrm{~mL}$ de água. $\mathrm{A}$ fração acetato de etila obtida foi transferida para um balão volumétrico e acetato de etila foi acrescentado qsp $25 \mathrm{~mL}$, obtendo-se assim a solução-mãe (SM).

Uma alíquota de $1 \mathrm{~mL}$ da SM foi adicionada a $0,4 \mathrm{~mL}$ da SRF e o volume final das soluções foram ajustados com ácido acético/metanol 5\% qsp $10 \mathrm{~mL}$. As absorvâncias das soluções obtidas foram lidas em espectrofotômetro a $425 \mathrm{mn}$ após 30 min. (Farmacopéia brasileira, 2001). O branco constou de $1 \mathrm{~mL} \mathrm{SM}$ acrescido de acético/metanol $5 \%$ qsp. $10 \mathrm{~mL}$.

Os resultados dos teores encontrados foram distribuídos na matriz de contrastes contida em uma macro de planejamento fatorial do software Excel (Teófilo \& Ferreira., 2006).

Ressalta-se que uma nova otimização foi realizada por meio univariado, após a definição das variáveis significativas, em três grupos diferentes $(n=6)$.

\section{Tratamentos dos dados}

Todos os dados foram avaliados por teste de rejeição (teste $Q$ ) e expressos em percentagem de padrão na droga seca, com intervalo de confiança (5\%), aplicando-se ANOVA e/ou Bonferroni e Tukey como análise estatística, no método univariado (5\%).

\section{RESULTADOS E DISCUSSÃO}

\section{Marchas fitoquímica}

Apesar de relatos populares das propriedades fitoterápicas do gênero Verbena (Morcelle et al., 2012), para a investigação científica de suas reais propriedades medicinais é necessário a determinação de seus principais componentes químicos.

As análises fitoquímicas têm como finalidade investigar os principais componentes químicos em espécies vegetais não estudadas. Caso o interesse seja restrito a uma classe, ou às substâncias responsáveis por atividades biológicas, a investigação deverá ser direcionada para o isolamento e elucidação estrutural das mesmas (Falkenberg et al., 2007).

A química dos componentes da espécie aqui analisada, v. minutiflora, é pouco estudada,

TABELA 2. Matriz de contrastes para o doseamento de flavonoides totais, com o planejamento fatorial $2^{4-1} \mathrm{com}$ ponto central.

\begin{tabular}{ccccc}
\hline Amostras & $\begin{array}{c}\text { Quantidade de } \\
\mathrm{HMT} 0,5 \%\end{array}$ & $\begin{array}{c}\text { Tipo de } \\
\text { ácido }\end{array}$ & $\begin{array}{c}\text { Volume } \\
\text { de ácido }\end{array}$ & $\begin{array}{c}\text { Tempo de } \\
\text { aquecimento }\end{array}$ \\
\hline $\mathbf{1}$ & $-(1 \mathrm{~mL})$ & $-(\mathrm{HCl})$ & $-(2 \mathrm{~mL})$ & $-(30$ min mais $3 \times 10 \mathrm{~min})$ \\
$\mathbf{2}$ & $+(2 \mathrm{~mL})$ & - & - & $+(15$ min mais $3 \times 5 \mathrm{~min})$ \\
$\mathbf{3}$ & - & $+\left(\mathrm{H}_{2} \mathrm{SO}_{4}\right)$ & - & + \\
$\mathbf{5}$ & + & + & - & - \\
$\mathbf{6}$ & - & - & $+(4 \mathrm{~mL})$ & - \\
$\mathbf{7}$ & + & + & + & - \\
$\mathbf{8}$ & + & + & + & + \\
Ponto central $\mathrm{n}=3$ & $1,5 \mathrm{~mL}$ & $\mathrm{HCl} \mathrm{e}_{2} \mathrm{SO}_{4}$ & $3 \mathrm{~mL} \mathrm{HCl} \mathrm{e} \mathrm{3} \mathrm{mL} \mathrm{H}_{2} \mathrm{SO}_{4}$ & 23 mais 8 min \\
\hline
\end{tabular}


trabalhos científicos a esse respeito são de grande importância, especialmente ante a promissoriedade de seu uso em fitoterapia.

Os resultados obtidos nas análises dos extratos aquosos e hidroalcoólicos da V. minutiflora encontram-se na Tabela 3.

A pesquisa de taninos foi positiva para 0 extrato aquoso (Tabela 3). Outros autores (Gutiérrez \& Villegas., 2008) também observaram a presença de taninos diante as reações com gelatina e cloreto férrico, nas partes aéreas de $V$. officinalis L., enquanto (Souza et al., 2005), em V. litoralis K. (partes aéreas), identificaram taninos catéquicos.

A análise de ácidos fixos foi positiva para o extrato aquoso (Tabela 3). Outros autores também relataram a presença desses metabólicos em espécies vegetais da família Verbenaceae onde, foram identificados em raízes de Stachytarpheta cayennensis (Rich.) Vahl. o ácido tetraeicosanóico (Vahl et al., 2010).

A presença de flavonoides foi positiva frente ao extrato hidroalcoólico (Tabela 3). Esse metabólito também foi detectado em análises fitoquímicas com outras espécies da família Verbenaceae, onde detectaram-se a presença de flavonoides em várias espécies, como em folhas, cascas dos caules e das raízes de Vitex gardneriana Schauer (Sá Barreto et al., 2007); nas partes aéreas, caules e folhas, de Priva lappulacea (L.) Pers (Tur et al., 2010); nos frutos de Duranta repens L. (Tur et al., 2010). Também foram relatadas a presença desses metabólitos nas partes aéreas de Verbena litoralis Kunth (Souza et al., 2005) ; (Li et al., 2003) e V. officinalis L. (Calvo., 2006), com isolamento, nos talos nessa última, de apigenina e luteolina
(Verma \& Siddiqui., 2011). Pesquisas realizadas com folhas e caule de Aloysia virgata (Ruiz \& Pav.) Juss. mostraram isolamento de luteolina (Vandresen et al., 2010). Em talos, raízes e folhas de Lippia sidoides Cham. encontrou-se naringenina, 3',4',5,7-tetra-hidroxiflavanona, 4',5,7-tri-hidroxi6-metoxiflavona, 2'-O-glicopiranosil-3,4,4',6'-tetrahidroxi-di-hidrochalcona e 2'-O-glicopiranosil-4,4',6'tri-hidroxi-di-hidrochalcona (Almeida et al., 2010) .

Os resultados analisados demonstraram ainda a presença de esteroides no extrato hidroalcoólico (Tabela 3). Outros estudos envolvendo espécies da família Verbenaceae, também evidenciaram a presença de triterpenos tais como o acetato do ácido oleanólico, olantandeno-A, a friedelinona e o acetato de 3-epifriedelanila (Vandresen et al., 2010). Há também relatos da presença de esteroides nessa família, como é o caso das misturas de $\beta$-sitosterol e estigmasterol em raízes e folhas de Lippia affinis gracillis H.B.K bem como nas folhas de Aloysia gratissima (Gillies \& Hook.) Tronc. onde encontraram-se os triterpenos: a-amirina, ácido betulínico, ácido oleanólico e ácido ursólico (Vandresen et al. , 2010).

Outro resultado importante foi a detecção de cumarinas no extrato hidroalcoólico (Tabela 3 ), dado esse respaldado pela literatura, onde outros trabalhos mostram a presença de cumarinas em outras espécies de Verbenaceaes, como no caso da Verbena litoralis Kunth (Souza et al., 2005 e Li et al., 2003). Outro grupo de metabólito secundário encontrado foi o de alcaloides no extrato hidroalcoólico (Tabela 3). Esses dados diferem dos dados encontrados em outras publicações de espécies da família Verbenaceae. A pesquisa

TABELA 3 Análise organoléptica e fitoquímica qualitativa dos extratos de $V$. minutiflora

\begin{tabular}{lcc}
\hline ENSAIOS & Extrato aquoso & Extrato Hidroalcoólico \\
\hline Cor & Mel & Mel \\
Odor & Adocicado & Adocicado \\
Sabor & amargo & amargo \\
pH & 5,87 & 5,85 \\
Heterosídeos antociânicos & - & $\mathrm{NR}$ \\
Saponinas & - & $\mathrm{NR}$ \\
Heterosídeos cianogenéticos & - & $\mathrm{NR}$ \\
Aminogrupos & + & + \\
Taninos & + & $\mathrm{NR}$ \\
Ácidos fixos & + & $\mathrm{NR}$ \\
Flavonoides & $\mathrm{NR}$ & + \\
Triterpenos & $\mathrm{NR}$ & + \\
Esteroides & $\mathrm{NR}$ & + \\
Alcaloides & $\mathrm{NR}$ & + \\
Cumarinas & $\mathrm{NR}$ & + \\
Antraquinonas & $\mathrm{NR}$ & - \\
\hline
\end{tabular}

NR: Não realizado ;”+": positivo; “-“ negativo

Rev. Bras. PI. Med., Campinas, v.18, n.1, p.1-8, 2016. 
desses metabólitos foi negativa em raízes de Lippia alba (Mill.) N.E. Brown (Filho et al., 2006); em folhas, cascas dos caules e das raízes de Vitex gardneriana Schauer (Sá Barreto et al., 2007) ou em partes aéreas de V. litoralis Kunth (Souza et al., 2005).

\section{Determinação de água}

A determinação do teor de água em vegetais in natura é de grande importância em ensaios que envolvam química de produtos naturais, pois está intimamente ligada com a conservação das drogas (Oliveira et al., 2005). As análises dos teores de água obtidos nas perdas por dessecações das flores de $\mathrm{V}$ minutiflora foram de $11,59 \pm 2,82 \%$. Os resultados evidenciados encontram-se dentro dos valores aceitáveis segundo literatura, uma vez que os valores de umidades estabelecidos para flores estão entre 8 a 14\% (Oliveira et al., 2005).

\section{Determinação da capacidade antioxidante}

Dentre os métodos mais utilizados para determinação da atividade antioxidante foi escolhido a utilização para se aplicar neste trabalho.

O método de determinação antioxidante escolhido, complexação com fosfomolibdênico, baseia-se na redução do molibdênio (VI) a molibdênio (V) com a formação de um complexo verde de fosfato/molibdênio $(V)$ na presença de substâncias com capacidade antioxidante (Prieto et al., 1999). O complexo desenvolvido demonstra ser compatível com diversos solventes orgânicos (metanol, etanol, dimetil sulfóxido, $n$-hexano); apresenta estabilidade à temperatura ambiente por várias horas; e é capaz de ser usado com padrões externos de diferentes polaridades, como o ácido ascórbico, BHT (butilhidroxitolueno), glutationa e tocoferois (Prieto et al., 1999)

\section{Curva analítica}

Foi confeccionada uma curva analítica utilizando-se como padrão externo o ácido L-ascórbico em sete diferentes concentrações. Obteve-se a equação linear, que foi expressa como $Y=7,727 \cdot 10^{3}$. $X-0,1410$ com o intervalo de $2,36 \cdot 10^{-5}$ a $1,8 \cdot 10^{-4} \mathrm{mmol} \cdot \mathrm{mL}^{-1}$ e $\mathrm{R}^{2}=0,99579$. Todos os parâmetros estiveram dentro do recomendado pela legislação brasileira (Brasil, 2003).
Os resultados obtidos com o planejamento fatorial $2^{4-1}$ para a determinação da capacidade antioxidante estão listados na planilha quimiométrica (Tabela. 4).

O planejamento fatorial $2^{4-1}$ indicou efeito de $1^{\mathrm{a}}$ ordem, uma variável significativa, para líquido extrator, método de extração e tempo de extração. Para o líquido extrator percebeu-se que ao diminuir a polaridade, ou seja, acrescentar etanol, a extração dos componentes antioxidantes é aumentada. $O$ tempo de extração maior (15 min) também aumenta significativamente a extração de antioxidantes. Já a alteração da extração com vórtex para a efetuada com sonicador, o teor de antioxidante é reduzido.

Como efeitos de $2^{\mathrm{a}}$ ordem, duas variáveis significativas, percebeu-se que o aumento do $\mathrm{pH}$ e extração por sonicador, leva à um aumento da quantidade de antioxidantes; o mesmo é notado quando se usa água:etanol 50:50 com o tempo de 15 min. Como a alteração de pH não foi significativa em nenhuma análise, escolheu-se utilizar o pH 1. Os níveis "tipos de extrações" (vórtex e sonicador) são qualitativos, assim, optou-se pelo uso de vórtex por apresentar efeito de $1^{\mathrm{a}}$ ordem.

Como as demais variáveis significativas são passíveis de otimizações, seguiu-se um novo planejamento fatorial $2^{2}$ (ponto central $n=3$ ) com as variáveis: líquido extrator (água:etanol 50:50 ou 20:80) e tempo de extração (15 ou $25 \mathrm{~min}$ ).

Observaram-se efeito de $1^{\text {a }}$ ordem em ambas as variáveis: com o aumento do tempo, a quantidade de antioxidantes extraída aumentou; com adição do etanol, a quantidade de antioxidantes foi reduzida.

Foi modificado o planejamento fatorial $2^{2}$, realizado com as variáveis tempo (25 ou $35 \mathrm{~min}$ ) e líquido extrator (água:etanol 80:20 ou 50:50). Somente o tempo de extração apresentou efeito significativo (de $1^{\text {a }}$ ordem), aumentando o teor de antioxidantes extraídos quando se elevou o tempo.

Em decorrência de apenas a variável tempo de extração foi significativa, a próxima otimização foi univariada com 35, 45 e 55 min, utilizando-se o preparo de amostra com água:etanol 50:50, pH 1 em vórtex. Não houve diferença significativa entre os tempos de extração $(p=0,23)$. Dessa forma, o melhor método após a extração dos antioxidantes

TABELA 4. Significância das variáveis do planejamento fatorial fracionário $2^{4-1}$ para determinação da capacidade antioxidante (desvio do efeito $=0,00835$ )

\begin{tabular}{lcl}
\hline Variável & Efeito calculado da variável & Resultado \\
\hline $\mathrm{pH}$ & 0,003055279 & Variável não significativa \\
Líquido extrator & 0,025028152 & Variável significativa, com melhores valores com etanol \\
Método de extração & $-0,013667354$ & Variável significativa, melhores resultados em vórtex \\
Tempo de extração & 0,010658415 & Variável significativa, com aumento em 15 min \\
$\mathrm{pH}+$ método de extração & 0,021336637 & Variável significativa \\
\hline
\end{tabular}

Rev. Bras. PI. Med., Campinas, v.18, n.1, p.1-8, 2016. 
de V. minutiflora. foi obtido por: extração em vórtex por $35 \mathrm{~min}$, com água:etanol 50:50 em pH 1, na qual a capacidade antioxidante pode ser expressa como $0,1899 \pm 5,8 \cdot 10^{-3} \mathrm{mmol}$ de ácido ascórbico.g- ${ }^{-1}$ de flores de $V$. minutiflora.

\section{Totais}

\section{Determinação do teor de Flavonoides}

Existem diversos métodos para o doseamento de flavonoides totais, desde técnicas espectrométricas até a utilização de CLAE, CCD ou $\mathrm{CG}$, entre outras. A metodologia do $\mathrm{AlCl}_{3}$ foi escolhida para a determinação espectrofotométrica in vitro de flavonoides totais nas flores de V. minutiflora por se tratar de uma técnica simples, com validação analítica (Grubešić et al., 2007); (Marcucci et al., 1988) e apresentar-se como um procedimento padrão para a determinação de flavonoides totais em espécies vegetais descritas em farmacopeias (Farmacopeia Brasileira, 2001); (Brasil, 2010b; 2010c). É válido lembrar ainda, que este método evidenciou ser eficaz para a identificação específica de flavonas e flavonois (Chang et al., 2002).

\section{Curva analítica}

A quercetina foi empregada em onze concentrações diferentes, obtendo-se uma equação expressa como $Y=0,0863 . x-0,00908$, no intervalo de 0,2-3,6 $\mu \mathrm{g} \cdot \mathrm{mL}^{-1}$, com $\mathrm{R}^{2}=0,99361$ - de acordo com o exigido pela legislação brasileira (Brasil, 2003).

\section{Preparo de amostra}

Os resultados obtidos com o planejamento fatorial 24-1 para a determinação do teor de flavonoides totais. No planejamento fatorial $2^{4-1}$, as variáveis significativas $\left(1^{\text {a }}\right.$ ordem) foram tipo de ácido e volume de ácido ( $p=0,019$ e 0,024, respectivamente).

Em relação ao uso de $\mathrm{HCl}(2 \mathrm{~mL})$, os resultados demonstraram maiores teores de flavonoides quando se utiliza este volume. Entretanto, em relação às demais variáveis testadas não foram encontradas diferenças significativas nos variados volumes de HMT testados ou com o tempo de aquecimento: nesse último caso, o método farmacopeico (Farmacopeia Brasileira, 2001)sugere 30 min, mas nossos resultados mostram que para essa amostra, pode ser utilizado o tempo de $15 \mathrm{~min}$.

Em virtude da variável "tipo de ácido" ser qualitativa, decidiu-se por realizar uma otimização univariada com o volume de $\mathrm{HCl}$ usado, em $1 \mathrm{~mL}$, $1,5 \mathrm{~mL}$ e $2 \mathrm{~mL}$, tendo definidos a quantidade de HMT a $1 \mathrm{~mL}$ e o tempo de aquecimento de $15 \mathrm{~min}$. Não ocorreu diferença significativa entre os volumes de $\mathrm{HCl}(p=0,77)$.

Assim, devido ao uso do planejamento fatorial pode-se observar que os níveis das variáveis "volume de ácido" e "tempo de aquecimento", conforme o método oficial (Farmacopeia Brasileira, 2001), podem ser alterados pelas condições otimizadas. O método de preparo de amostra demonstrou que o uso de $1 \mathrm{ml}$ de $\mathrm{HCl}$ e $15 \mathrm{~min}$ mais três vezes de $5 \mathrm{~min}$, sendo respectivamente, $\mathrm{o}$ volume de ácido e o tempo de aquecimento, é uma melhor forma de determinar o teor de flavonoides totais.

Esse resultado gera a redução do volume de ácido usado e do tempo de aquecimento sob refluxo, obtendo-se igualmente o composto de interesse sem nenhuma interferência nos resultados encontrados, podendo assim, serem substituídos pelas condições descritas no método farmacopeico.

Partindo-se do preparo de amostra com $1 \mathrm{~mL}$ de $\mathrm{HCl}$ e $1 \mathrm{~mL}$ de HMT por um tempo de aquecimento de $15 \mathrm{~min}$, foi obtido um teor de flavonoides totais equivalente a $6,748.10^{-2} \pm$ $2,085.10^{-3} \%$ expressos em quercetina nas flores de $V$. minutiflora.

\section{CONCLUSÃO}

A marcha fitoquímica realizada com flores de $V$. minutiflora demonstrou a presença de grupos de metabólitos especiais, tais como os taninos, ácidos fixos, flavonoides, esteroides, e/ou triterpenos, cumarinas, alcaloides e aminogrupos.

Os resultados aqui obtidos demonstram que o uso de planejamento fatorial na realização de experimentos fitoquímicos apresenta-se como um instrumento eficaz para a otimização dos resultados encontrados. Demonstrou-se através dele que o melhor método de extração dos componentes antioxidantes, de flores de $V$. minutiflora é o que utiliza o vórtex por $35 \mathrm{~min}$, água:etanol 50:50 em pH 1 como solvente. Obteve-se após a extração otimizada uma capacidade antioxidante de 0,1899 $\pm 5,8.10^{-3} \mathrm{mmol}$ expressos em ácido ascórbico. $\mathrm{g}^{-1} \mathrm{de}$ flores de $V$. minutiflora.

O PF demonstrou que na determinação do teor de flavonoides totais, o melhor método de preparo de amostra foi obtido com o uso de $1 \mathrm{~mL}$ de $\mathrm{HCl}$ e $1 \mathrm{~mL}$ de HMT, por um tempo de aquecimento sob refluxo de 15 min mais $5 \mathrm{~min}$; onde forneceu $6,748.10^{-2} \pm 2,085.10^{-3} \%$ expressos em quercetina nas flores de $V$. minutiflora.

\section{REFERÊNCIAS}

ALMEIDA, M.C.S. DE; et al.,Flavonoides e outras substâncias de Lippia sidoides e suas atividades antioxidantes. Química Nova, v. 33, n. 9, p. 1877-1881, 2010.

BRASIL. AGÊNCIA NACIONAL DE VIGILÂNCIA SANITÁRIA. Determinação de água em drogas vegetais. Farmacopeia Brasileira, 5. ed., v. 1, Brasília:

Rev. Bras. PI. Med., Campinas, v.18, n.1, p.1-8, 2016. 
ANVISA, 2010a, p. 197.

BRASIL. AGÊNCIA NACIONAL DE VIGILÂNCIA SANITÁRIA. Abacateiro. Farmacopeia Brasileira, 5. ed. v. 2. Brasília: Anvisa, 2010b, p. 554-555.

BRASIL. AGÊNCIA NACIONAL DE VIGILÂNCIA SANITÁRIA. Calêndula. Farmacopeia Brasileira, 5. ed. v. 2. Brasília: Anvisa, 2010c, p. 712-715.

BRASIL, Resolução RE n 899, 29 de maio de 2003. Guia para a validação de métodos analíticos e bioanalíticos. D.O.U. - Diário Oficial da União; poder executivo, de 02 de junho de 2003

CALVO, M.I. Anti-inflammatory and analgesic activity of the topical preparation of Verbena officinalis L. Journal of Ethnopharmacology, v. 107, n. 3, p. 380-2, 2006.

CHANG, C; et al.,Estimation of total flavonoid content in propolis by two complementary colorimetric methods. Journal of Food and Drug Analysis, v. 10, n. 3, p. 178-182, 2002

FALKENBERG, M.B; SANTOS, R.I; SIMÕES, C.M.O. Introdução à análise fitoquímica. In: SIMÕES, C; SCHENCKEL, E; GOSMANN, G; MELLO, J; MENTZ, L; PETROVICK, P. (Org.). Farmacognosia - da planta ao medicamento. 6. ed. Porto Alegre/Florianópolis: editora da UFRGS/ Editora da UFSC, 2007, p. 229-245.

FARMACOPEIA Brasileira. Macela. Farmacopeia Brasileira. 4. ed., Parte II, Fasc. 3, São Paulo: Atheneu, 2001, p. 158-1 - 158-7.

FILHO, J.G.Set al., . Antimicrobial activity and phytochemical profile from the roots of Lippia alba (Mill.) N.E. Brown. Brazilian Journal of Pharmacognosy, v. 16, n. 4, p. 506-509, 2006.

GRUBEŠIĆ, R.J.; et al., Flavonoid Content Assay: Prevalidation and Application on Plantago L. Species, Acta Chimica Slovenica. v. 54, p. 397-406, 2007.

GUTIÉRREZ, G.P.A.; VILLEGAS, M.C.V. Efecto tóxico de Verbena officinalis (família Verbenaceae ) en Sitophilus granarius (coleoptera: curculionidae). Revista Lasallista de Investigación, v. 5, n. 2, p. 74-82, 2008.

LAMEIRA, O. A.; PEREIRA PINTO, J. E. B. Plantas Medicinais: do cultivo, manipulação e uso à recomendação popular. Embrapa Amazônia Oriental, p. 21-24, 2008.

LI, Y.; et al., Sterol and triterpenoid constituents of Verbena littoralis with NGF-potentiating activity. Journal of Natural Products, v. 66, n. 5, p. 696-8, 2003.

MACIEL, M.A.M; et al., Plantas medicinais: a necessidade de estudos multidisciplinares. Química. Nova, v. 25, n. 3, p. 429-438, 2002.

MARCUCCI, M.C.; et al., A. Uso de cloreto de alumínio na quantificação de flavonoides em amostras de própolis. Mensagem Doce, v.46, p. 3-8, 1988.

MORCELLE, M.R.; GATTUSO, M.A; APÓSTOLO,M.M.
Stem and leaf anatomy of six species of Verbena native to Buenos Aires province, Argentina. New Zealand Journal of Botany. v.50, n1, p.3-14, 2012.

MATOS, F.J. A. Introdução à Fitoquímica Experimental. 2. ed. Fortaleza: Edições UFC, 1997, 141p.

OLIVEIRA, F.;AKISUE, G.; AKISUE, M.K. Farmacognosia. São Paulo: Atheneu, 1.ed. 2005, p. 424.

PRIETO, P.; et al., Spectrophotometric quantitation of antioxidant capacity through the formation of a phosphomolybdenum complex: specific application to the determination of vitamin E. Analytical Biochemistry, v. 269, n. 2, p. 337-41, 1999.

SÁ BARRETO, L.C.L.; et al., Atividade Moluscicida de Extratos e de Aucubina de Vitex gardneriana Schauer (Verbenaceae ) em Embriões da Biomphalaria glabrata. Latin American Journal of Pharmacy, v. 26, n. 3, p. 339-343, 2007.

SILVA, J.A.; et al.,Influência das Variáveis Nitrogênio, Fósforo e Brix na Produção dos Metabólitos Secundários Contaminantes Totais da Fermentação Alcoólica. Química Nova, v. 29, n. 4, p. 695-698, 2006.

SOUZA, T.J.T.; et al., Análise Morfo-Histológica e Fitoquímica de Verbena litoralis Kunth. Acta Farmacêutica. Bonaerense, v. 24, n. 2, p. 209-214, 2005.

SOUZA, C. D.; FELFILI, J. M. Uso de plantas medicinais na região de Alto Paraíso de Goiás, GO, Brasil. Acta Botanica Brasilica. v. 20, n. 1, p.135-142, 2006.

TEÓFILO, R.F.; FERREIRA, M.M. C. Quimiometria ii: planilhas eletrônicas para cálculos de planejamentos experimentais, um tutorial. Química Nova, v. 29, n. 2, p. 338-350, 2006.

TUR, C.M.; et al., Alelopatia de extratos aquosos de Duranta repens sobre a germinação e o crescimento inicial de Lactuca sativa e Lycopersicum esculentum. Biotemas, v. 23, n. 2, p. 13-22, 2010.

VAHL, R.; et al., Evaluation of antimicrobial , antioxidant and phototoxic activities of extracts and isolated compounds from Stachytarpheta cayennensis. Brazilian Journal of Pharmacognosy, v. 20, n. 6, p. 922-928, 2010.

VANDRESEN, F.; et al.,. Constituintes químicos e avaliação das atividades antibacteriana e antiedematogênica de Aloysia gratissima (Gillies \& Hook.) Tronc. e Aloysia virgata (Ruiz \& Pav.) Pers., Verbenaceae. Brazilian Journal of Pharmacognosy, v. 20, n. 3, p. 317-321, 2010.

VERMA, V.K.; SIDDIQUI, N.U. Bioactive chemical constituents from the plant Verbena officinalis linn. International Journal of Pharmacy and Pharmaceutical Sciences, v. 3, n. 4, p. 108-109, 2011.

Rev. Bras. PI. Med., Campinas, v.18, n.1, p.1-8, 2016. 